\title{
Effect of Various Organic Manures with Biofertilizers on Growth, Yield and Economics of Onion (Allium cepa L.)
}

\author{
Kalpana Nirala, Shailaja Punetha*, S.C. Pant and Sandeep Upadhaya \\ Department of Vegetable Science, ${ }^{3}$ Department of Soil science, College of Horticulture, VCSG \\ UUHF, Bharsar, PauriGarhwal, Uttarakhand, India-246123 \\ *Corresponding author
}

\section{A B S T R A C T}

Keywords

Biofertilizer, Growth, Yield, Economics, Onion, Organic manure

Article Info

\section{Accepted:}

26 December 2018

Available Online:

10 January 2019
An experiment was conducted at the Organic Production Block, College of Horticulture, VCSG Uttarakhand University of Horticulture and Forestry, Bharsar, Uttarakhand during the year 2016. The experiment was laid out in randomized block design with three replicated and 9 treatments viz., $\mathrm{T}_{1}$ Control, $\mathrm{T}_{2}$ Vermicompost, $\mathrm{T}_{3} \mathrm{FYM}, \mathrm{T}_{4}$ Goat manure, $\mathrm{T}_{5}$ Neem cake, $\mathrm{T}_{6}$ Vermicompost $+\mathrm{FYM}+$ Goat manure, $\mathrm{T}_{7}$ Vermicompost $+\mathrm{FYM}+$ Neem cake, $\mathrm{T}_{8}$ Vermicompost + Goat manure + Neem cake, T9 FYM + Goat manure + Neem cake. The observations were recorded on different growth and yield attributes. Further, economics of different treatments was also worked out. The analysis of variance revealed significant differences among the treatments for all the characters under study. The treatment T3 FYM due to its persistent performance for yield (324.46 q/ha), gross income (Rs. 324460.00 q/ha), net returns (Rs. 251680.04 q/ha) and higher benefit: cost ratio (1:3.45). Thus, it can be concluded from the findings that application of FYM $\left(2.8 \mathrm{~kg} / 1.2 \mathrm{~m}^{2}\right)$ can be recommended for commercial cultivation of onion.

\section{Introduction}

Onion (Allium cepa L.) is one of the most important commercial vegetable crops cultivated extensively in India and belongs to the family Alliaceae and originated from the central Asia (Thamburaj and Singh, 2001). It is an indispensable item in every kitchen as vegetable and condiment. It is one of the few versatile vegetable crops that can be kept for a fairly long period and can safely with stand the hazards of rough handling long distance transport. Organic manures are considered helpful in improving the physical and nutritional status of the soil and also enhance the activity of soil microflora. They also add considerable amount of major nutrients in the soil besides, improving the soil properties. Further, decomposition of organics in the soil leads to different types of biological reactions which are helpful in preventing various diseases causing pathogens (Ramesh et al., 2010). Biofertilizers offer an economically attractive and ecologically sound means of reducing external inputs and improving quality and quantity of vegetable produce. They contain microorganisms which are capable of mobilizing nutrient elements from 
unavailable form to available form through different biological processes.

\section{Materials and Methods}

The present investigation was carried out at the Organic Production Block, college of Horticulture and Forestry, Bharsar, Uttarakhand during the Year 2016 "Agrifound Light Red" cultivar of onion. The experiment was laid out in a randomized complete block design (RCBD) at spacing $15 \mathrm{~cm} \times 10 \mathrm{~cm}$ and plot size $1.20 \times 1.0 \mathrm{~m}^{2}$ with 3 replications and 10 treatments, which consisted of sole application of organic sources (Vermicompost, FYM, Goat manure, Neem cake)and their combinations (Table 1). In all treatments planting material were dipped in mixed culture of biofertilizers (Azotobacter + PSB). The data were recorded on growth and yield parameters like plant height $(\mathrm{cm})$, number of leaves per plant, leaf length $(\mathrm{cm})$, leaf diameter $(\mathrm{cm})$, root length $(\mathrm{cm})$, number of roots per plant, bulb length $(\mathrm{cm})$, polar diameter $(\mathrm{cm})$, equatorial diameter $(\mathrm{cm})$, neck length $(\mathrm{cm})$, neck thickness $(\mathrm{cm})$, number of scales per bulb, bulb weight $(\mathrm{g})$ yield per hectare (q/ha). Total soluble solid $\left({ }^{0} \mathrm{~B}\right)$ and cost: benefit ratio and mean data were subjected to statistically analysis as per Gomez and Gomez (1984). Further; economics analysis of different treatments was tested depending upon the locally existing fixed and variable costs of different inputs.

\section{Results and Discussion}

\section{Growth characters}

The data pertaining to different growth characters at different stages revealed significant variations among the different treatments under study (Table 2 and 3). Maximum plant height $(25.30 \mathrm{~cm}, 46.46 \mathrm{~cm}$, and $56.93 \mathrm{~cm}$ ), number of leaves per plant (5.45, 7.46 and 9.16), root length $(7.50 \mathrm{~cm}$, $8.18 \mathrm{~cm}$ and $10.50 \mathrm{~cm}$ ), and number of roots per plant $(28.02,70.78$ and 107.89) was recorded at different stages 50,100 and 150 days after transplanting by the application of Vermicompost $\left(\mathrm{T}_{2}\right)$.This might due to application of vermicompost may be due to its rich content of macro and micro nutrients, vitamins, growth hormones and micro flora Bhavalkar (1991).

Table.1 Detail of treatments used in the present study

\begin{tabular}{|l|l|}
\hline $\begin{array}{c}\text { Treatment } \\
\text { Code }\end{array}$ & \multicolumn{1}{c|}{ Treatment Detail } \\
\hline $\mathbf{T}_{\mathbf{1}}$ & Control \\
\hline $\mathbf{T}_{\mathbf{2}}$ & Vermicompost @ 0.6 kg/plot \\
\hline $\mathbf{T}_{\mathbf{3}}$ & FYM @ 2.8kg / plot \\
\hline $\mathbf{T}_{\mathbf{4}}$ & Goat manure @ 0.5 kg / plot \\
\hline $\mathbf{T}_{\mathbf{5}}$ & Neem cake @ 0.3 kg / plot \\
\hline $\mathbf{T}_{\mathbf{6}}$ & $\begin{array}{l}\text { Vermicompost + FYM + Goat manure complex } \\
\left(1 / 3^{\text {rd }} \text { amount of each i.e. @ 0.2;0.93 and 0.16 kg/plot }\right.\end{array}$ \\
\hline $\mathbf{T}_{\mathbf{7}}$ & $\begin{array}{l}\text { Vermicompost + FYM + Neem cake complex } \\
\left(1 / 3^{\text {rd }} \text { amount of each i.e. @ 0.2;0.93 and } 0.1 \mathrm{~kg} / \mathrm{plot}\right)\end{array}$ \\
\hline $\mathbf{T}_{\mathbf{8}}$ & $\begin{array}{l}\text { Vermicompost + Goat manure + Neem cake complex } \\
\left(1 / 3^{\text {rd }} \text { amount of each i.e. @ 0.2;0.16 and } 0.1 \mathrm{~kg} / \mathrm{plot}\right)\end{array}$ \\
\hline $\mathbf{T}_{\mathbf{9}}$ & $\begin{array}{l}\text { FYM + Goat manure + Neem cake complex } \\
\left(1 / 3^{\text {rd }} \text { amount of each i.e. @ 0.93;0.16 and } 0.1 \mathrm{~kg} / \mathrm{plot}\right)\end{array}$ \\
\hline
\end{tabular}


Table.2 Effect of various organic manures with biofertilizers and their combinations on plant height, numbers of leaves per plant and Leaf length at different stages

\begin{tabular}{|c|c|c|c|c|c|c|c|c|c|}
\hline \multirow[t]{2}{*}{$\begin{array}{l}\text { Treatment } \\
\text { code }\end{array}$} & \multicolumn{3}{|c|}{ Plant height $(\mathrm{cm}) \pm \mathrm{SE}(\mathrm{m})$} & \multicolumn{3}{|c|}{$\begin{array}{l}\text { Number of leaves per } \\
\text { plant }(\mathrm{cm}) \pm \mathrm{SE}(\mathrm{m})\end{array}$} & \multicolumn{3}{|c|}{ Leaf length $(\mathrm{cm}) \pm \mathrm{SE}(\mathrm{m})$} \\
\hline & 50 DAT & $\begin{array}{c}100 \\
\text { DAT }\end{array}$ & $\begin{array}{c}150 \\
\text { DAT }\end{array}$ & 50 DAT & $\begin{array}{c}100 \\
\text { DAT }\end{array}$ & $\begin{array}{c}150 \\
\text { DAT }\end{array}$ & 50 DAT & $\begin{array}{c}100 \\
\text { DAT }\end{array}$ & $\begin{array}{l}150 \\
\text { DAT }\end{array}$ \\
\hline $\mathbf{T}_{1}$ & $\begin{array}{c}15.57 \pm \\
0.48\end{array}$ & $\begin{array}{c}30.10 \pm \\
0.43\end{array}$ & $\begin{array}{c}39.97 \pm \\
0.44\end{array}$ & $\begin{array}{c}3.10 \pm \\
0.23\end{array}$ & $\begin{array}{c}5.16 \pm \\
0.40\end{array}$ & $\begin{array}{l}7.21 \pm \\
0.06\end{array}$ & $\begin{array}{c}12.24 \pm \\
0.15\end{array}$ & $\begin{array}{c}26.66 \pm \\
0.16\end{array}$ & $\begin{array}{c}36.31 \pm \\
0.16\end{array}$ \\
\hline $\mathbf{T}_{2}$ & $\begin{array}{c}25.30 * \pm \\
0.33\end{array}$ & $\begin{array}{c}46.46^{*} \pm \\
0.74\end{array}$ & $\begin{array}{l}56.93^{*} \pm \\
3.32\end{array}$ & $\begin{array}{l}5.45^{*} \pm \\
0.03\end{array}$ & $\begin{array}{c}7.46^{*} \pm \\
0.06\end{array}$ & $\begin{array}{c}9.16 * \pm \\
0.03\end{array}$ & $\begin{array}{c}20.64 * \pm \\
0.28\end{array}$ & $\begin{array}{l}40.46 * \pm \\
0.17\end{array}$ & $\begin{array}{l}51.27 * \pm \\
0.02\end{array}$ \\
\hline $\mathbf{T}_{3}$ & $\begin{array}{c}20.94 * \pm \\
0.31\end{array}$ & $\begin{array}{l}41.30 * \pm \\
0.67\end{array}$ & $\begin{array}{l}51.66 * \pm \\
0.63\end{array}$ & $\begin{array}{l}5.34 * \pm \\
0.36\end{array}$ & $\begin{array}{c}7.22 * \pm \\
0.01\end{array}$ & $\begin{array}{l}8.39 * \pm \\
0.02\end{array}$ & $\begin{array}{c}17.61 *_{ \pm} \\
0.01\end{array}$ & $\begin{array}{l}38.63^{*} \pm \\
0.12\end{array}$ & $\begin{array}{l}49.66 * \pm \\
0.08\end{array}$ \\
\hline $\mathbf{T}_{4}$ & $\begin{array}{l}20.68 * \pm \\
0.74\end{array}$ & $\begin{array}{l}34.78 * \pm \\
1.75\end{array}$ & $\begin{array}{c}50.48^{*} \pm \\
0.61\end{array}$ & $\begin{array}{c}4.47 * \pm \\
0.10\end{array}$ & $\begin{array}{l}7.23 * \pm \\
0.03\end{array}$ & $\begin{array}{l}8.23 * \pm \\
0.03\end{array}$ & $\begin{array}{c}16.34 * \pm \\
0.19\end{array}$ & $\begin{array}{c}32.44 * \pm \\
0.10\end{array}$ & $\begin{array}{l}47.48 * \pm \\
0.10\end{array}$ \\
\hline $\mathbf{T}_{5}$ & $\begin{array}{c}20.23^{*} \pm \\
0.03\end{array}$ & $\begin{array}{c}39.62 * \pm \\
0.14\end{array}$ & $\begin{array}{c}48.09 * \pm \\
0.13\end{array}$ & $\begin{array}{l}3.93 \pm \\
0.66\end{array}$ & $\begin{array}{c}7.21 * \pm \\
0.00\end{array}$ & $\begin{array}{l}7.46 * \pm \\
0.03\end{array}$ & $\begin{array}{l}18.23^{*} \\
\pm 0.03\end{array}$ & $\begin{array}{c}35.62 * \pm \\
0.14\end{array}$ & $\begin{array}{l}39.42 * \pm \\
0.24\end{array}$ \\
\hline $\mathbf{T}_{6}$ & $\begin{array}{c}21.83^{*} \pm \\
0.11\end{array}$ & $\begin{array}{c}37.02 * \pm \\
0.30\end{array}$ & $\begin{array}{c}48.68 * \pm \\
0.59\end{array}$ & $\begin{array}{c}3.55 \pm \\
0.17\end{array}$ & $\begin{array}{c}6.46^{*} \pm \\
0.46\end{array}$ & $\begin{array}{c}7.23 \pm \\
0.04\end{array}$ & $\begin{array}{c}19.50 \\
* \pm 0.25\end{array}$ & $\begin{array}{c}34.36 * \pm \\
0.06\end{array}$ & $\begin{array}{c}43.68^{*} \pm \\
0.03\end{array}$ \\
\hline $\mathbf{T}_{7}$ & $\begin{array}{c}23.16^{*} \pm \\
0.54\end{array}$ & $\begin{array}{c}45.81 * \pm \\
0.48\end{array}$ & $\begin{array}{c}50.30 * \pm \\
0.56\end{array}$ & $\begin{array}{c}5.26^{*} \pm \\
0.04\end{array}$ & $\begin{array}{c}7.26^{*} \pm \\
0.03\end{array}$ & $\begin{array}{c}9.07 * \pm \\
0.06\end{array}$ & $\begin{array}{c}22.49 * \pm \\
0.24\end{array}$ & $\begin{array}{c}42.48^{*} \pm \\
0.16\end{array}$ & $\begin{array}{c}49.30 * \pm \\
0.12\end{array}$ \\
\hline $\mathbf{T}_{8}$ & $\begin{array}{c}20.97 * \pm \\
0.07\end{array}$ & $\begin{array}{c}43.65^{*} \pm \\
0.21\end{array}$ & $\begin{array}{c}49.41 * \pm \\
0.27\end{array}$ & $\begin{array}{c}4.99 * \pm \\
0.39\end{array}$ & $\begin{array}{c}6.72 * \pm \\
0.03\end{array}$ & $\begin{array}{c}8.02 * \pm \\
0.03\end{array}$ & $\begin{array}{c}18.64 * \pm \\
0.26\end{array}$ & $\begin{array}{c}39.65^{*} \pm \\
0.21\end{array}$ & $\begin{array}{c}45.74 * \pm \\
0.11\end{array}$ \\
\hline $\mathbf{T}_{9}$ & $\begin{array}{c}22.81 *^{*} \pm \\
0.50\end{array}$ & $\begin{array}{c}41.67 * \pm \\
0.33\end{array}$ & $\begin{array}{c}50.23 * \pm \\
0.03\end{array}$ & $\begin{array}{c}5.43 * \pm \\
0.09\end{array}$ & $\begin{array}{c}7.27 * \pm \\
0.39\end{array}$ & $\begin{array}{c}9.05^{*} \pm \\
0.01\end{array}$ & $\begin{array}{c}16.81 * \pm \\
0.09\end{array}$ & $\begin{array}{c}39.34 * \pm \\
0.02\end{array}$ & $\begin{array}{c}47.23 * \pm \\
0.03\end{array}$ \\
\hline$\pm \mathrm{SE}(\mathrm{d})$ & 0.51 & 1.03 & 1.68 & 0.42 & 0.35 & 0.029 . & 0.21 & 0.18 & 0.17 \\
\hline $\mathrm{CD}_{(0.05)}$ & 1.10 & 2.20 & 3.60 & 0.91 & 0.75 & 0.062 & 0.46 & 0.38 & 0.37 \\
\hline
\end{tabular}

Significant at $5 \%$ level respectively 
Table.3 Effect of various organic manures with biofertilizers and their combinations on leaf diameter, root length and number of roots per plant at different stages

\begin{tabular}{|c|c|c|c|c|c|c|c|c|c|}
\hline \multirow[t]{2}{*}{$\begin{array}{l}\text { Treatment } \\
\text { code }\end{array}$} & \multicolumn{3}{|c|}{ Leaf diameter $(\mathrm{cm}) \pm \mathrm{SE}(\mathrm{m})$} & \multicolumn{3}{|c|}{ Root length $(\mathrm{cm}) \pm \mathrm{SE}(\mathrm{m})$} & \multicolumn{3}{|c|}{$\begin{array}{l}\text { Number of roots per plant } \\
(\mathrm{cm}) \pm \mathrm{SE}(\mathrm{m})\end{array}$} \\
\hline & 50 DAT & $\begin{array}{l}\text { 100 } \\
\text { DAT }\end{array}$ & $\begin{array}{l}150 \\
\text { DAT }\end{array}$ & 50 DAT & $\begin{array}{l}\text { 100 } \\
\text { DAT }\end{array}$ & 150 DAT & 50 DAT & $\begin{array}{l}100 \\
\text { DAT }\end{array}$ & 150 DAT \\
\hline $\mathbf{T}_{1}$ & $\begin{array}{c}0.53 \pm \\
0.06\end{array}$ & $\begin{array}{c}0.68 \pm \\
0.03\end{array}$ & $\begin{array}{c}0.88 \pm \\
0.03\end{array}$ & $\begin{array}{c}5.10 \pm \\
0.26\end{array}$ & $\begin{array}{c}7.11 \pm \\
0.23\end{array}$ & $\begin{array}{c}8.15 \pm \\
0.12\end{array}$ & $\begin{array}{c}20.12 \pm \\
0.31\end{array}$ & $\begin{array}{c}42.67 \pm \\
1.49\end{array}$ & $\begin{array}{c}80.63 \pm \\
0.29\end{array}$ \\
\hline $\mathbf{T}_{2}$ & $\begin{array}{c}0.67 \pm \\
0.03\end{array}$ & $\begin{array}{c}0.72 \pm \\
0.00\end{array}$ & $\begin{array}{l}1.31 \pm \\
0.65\end{array}$ & $\begin{array}{c}7.50 * \pm \\
0.13\end{array}$ & $\begin{array}{c}8.18^{*} \pm \\
0.14\end{array}$ & $\begin{array}{l}10.50 * \pm \\
0.02\end{array}$ & $\begin{array}{l}28.02 * \pm \\
0.25\end{array}$ & $\begin{array}{c}70.78 * \pm \\
0.33\end{array}$ & $\begin{array}{c}107.89 * \pm \\
1.69\end{array}$ \\
\hline $\mathbf{T}_{3}$ & $\begin{array}{c}0.73 \pm \\
0.03\end{array}$ & $\begin{array}{c}0.92 \pm \\
0.03\end{array}$ & $\begin{array}{l}1.41^{*} \pm \\
0.03\end{array}$ & $\begin{array}{l}6.91 * \pm \\
0.42\end{array}$ & $\begin{array}{c}7.13 \pm \\
0.54\end{array}$ & $\begin{array}{c}9.38 * \pm \\
0.03\end{array}$ & $\begin{array}{c}25.25 * \pm \\
0.54\end{array}$ & $\begin{array}{l}68.69 * \pm \\
0.86\end{array}$ & $\begin{array}{l}104.15^{*} \pm \\
9.55\end{array}$ \\
\hline $\mathbf{T}_{4}$ & $\begin{array}{c}0.77 \pm \\
0.03\end{array}$ & $\begin{array}{c}0.93 \pm \\
0.03\end{array}$ & $\begin{array}{c}1.01 \pm \\
0.03\end{array}$ & $\begin{array}{c}6.41 * \pm \\
0.33\end{array}$ & $\begin{array}{c}8.16 * \pm \\
0.14\end{array}$ & $\begin{array}{c}10.15 * \pm \\
0.03\end{array}$ & $\begin{array}{c}24.33^{*} \pm \\
0.57\end{array}$ & $\begin{array}{c}58.35 * \pm \\
8.95\end{array}$ & $\begin{array}{c}100.92 * \pm \\
0.03\end{array}$ \\
\hline $\mathbf{T}_{5}$ & $\begin{array}{c}0.58 \pm \\
0.03\end{array}$ & $\begin{array}{c}0.87 \pm \\
0.03\end{array}$ & $\begin{array}{c}0.90 \pm \\
0.03\end{array}$ & $\begin{array}{c}6.40 * \pm \\
0.03\end{array}$ & $\begin{array}{c}7.51 \pm \\
0.03\end{array}$ & $\begin{array}{c}9.80 * \pm \\
0.23\end{array}$ & $\begin{array}{c}23.49 * \pm \\
0.81\end{array}$ & $\begin{array}{l}52.67 \pm \\
3.83\end{array}$ & $\begin{array}{c}99.26^{*} \pm \\
0.64\end{array}$ \\
\hline $\mathbf{T}_{6}$ & $\begin{array}{c}0.61 \pm \\
0.03\end{array}$ & $\begin{array}{c}0.80 \pm \\
0.03\end{array}$ & $\begin{array}{c}0.93 \pm \\
0.03\end{array}$ & $\begin{array}{c}5.68 \pm \\
0.06\end{array}$ & $\begin{array}{c}6.40 \pm \\
0.02\end{array}$ & $\begin{array}{r}9.4 \\
0 .\end{array}$ & $\begin{array}{r}24.6 \\
0 .\end{array}$ & $\begin{array}{c}48.91 \pm \\
0.29\end{array}$ & $\begin{array}{l}94.87 * \pm \\
2.72\end{array}$ \\
\hline $\mathbf{T}_{7}$ & $\begin{array}{c}0.79 * \pm \\
0.31\end{array}$ & $\begin{array}{c}0.97 \pm \\
0.66\end{array}$ & $\begin{array}{c}1.61 * \pm \\
0.03\end{array}$ & $\begin{array}{c}7.05 * \pm \\
0.33\end{array}$ & $\begin{array}{c}7.82 \pm \\
0.09\end{array}$ & $\begin{array}{c}10.14^{*} \pm \\
0.02\end{array}$ & $\begin{array}{c}25.76 * \pm \\
1.21\end{array}$ & $\begin{array}{c}62.45^{*} \pm \\
3.53\end{array}$ & $\begin{array}{c}102.00 \\
1.66\end{array}$ \\
\hline $\mathbf{T}_{8}$ & $\begin{array}{c}0.74 \pm \\
0.02\end{array}$ & $\begin{array}{c}0.92 \pm \\
0.03\end{array}$ & $\begin{array}{c}1.05 \pm \\
0.01\end{array}$ & $\begin{array}{c}7.16^{*} \pm \\
0.03\end{array}$ & $\begin{array}{c}7.62 \pm \\
0.34\end{array}$ & $\begin{array}{c}8.91 * \pm \\
0.04\end{array}$ & $\begin{array}{c}21.05 \pm \\
0.59\end{array}$ & $\begin{array}{c}49.71 \pm \\
0.31\end{array}$ & $\begin{array}{c}99.44 * \pm \\
0.31\end{array}$ \\
\hline $\mathbf{T}_{9}$ & $\begin{array}{c}0.75 \pm \\
0.03\end{array}$ & $\begin{array}{c}0.96 \pm \\
0.03\end{array}$ & $\begin{array}{c}1.21 \pm \\
0.03\end{array}$ & $\begin{array}{c}7.20 * \pm \\
0.57\end{array}$ & $\begin{array}{c}7.38 \pm \\
0.31\end{array}$ & $\begin{array}{c}9.41 * \pm \\
0.06\end{array}$ & $\begin{array}{c}22.43 \pm \\
0.59\end{array}$ & $\begin{array}{c}52.27 \pm \\
1.61\end{array}$ & $\begin{array}{c}106.74 * \pm \\
0.30\end{array}$ \\
\hline$\pm \mathrm{SE}(\mathrm{d})$ & 0.12 & 0.17 & 0.21 & 0.40 & 0.41 & 0.18 & 0.89 & 5.23 & 4.89 \\
\hline $\mathrm{CD}_{(0.05)}$ & 0.25 & 0.33 & 0.60 & 0.87 & 0.88 & 0.40 & 1.91 & 11.19 & 10.45 \\
\hline
\end{tabular}

Significant at $5 \%$ level respectively 
Table.4 Effect of various organic manures with biofertilizers and their combinations on bulb length, polar diameter, equatorial diameter, neck length, neck thickness, number of scales per bulb, bulb weight, yield per hectare and total soluble solids at maturity

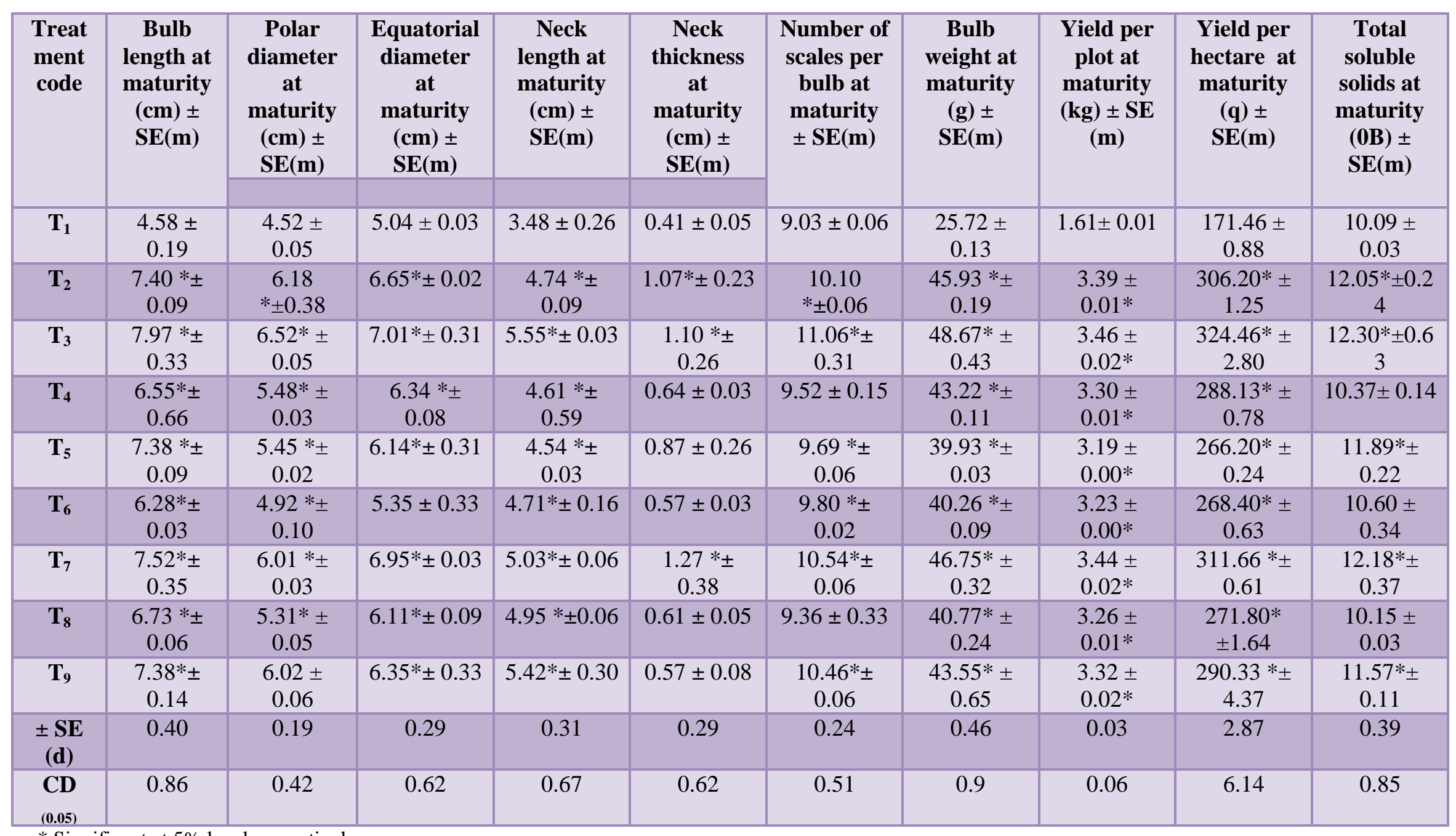

* Significant at 5\% level respectively 
Table.5 Effect of organic manure with biofertilizers and their combinations on economics of different treatments

\begin{tabular}{|c|c|c|c|c|c|}
\hline $\begin{array}{c}\text { Treatm } \\
\text { ent } \\
\text { code }\end{array}$ & $\begin{array}{c}\text { Yield } \\
\text { q/ha }\end{array}$ & $\begin{array}{c}\text { Total cost of } \\
\text { cultivation } \\
\text { (Rs/ha) }\end{array}$ & $\begin{array}{c}\text { Gross } \\
\text { Income } \\
\text { (Rs./ha) }\end{array}$ & $\begin{array}{c}\text { Net return } \\
\text { (Rs./ha) }\end{array}$ & C:B Ratio \\
\hline $\mathbf{T}_{\mathbf{1}}$ & 171.46 & 59195.15 & 171460.00 & 112264.85 & $1: 1.80$ \\
\hline $\mathbf{T}_{\mathbf{2}}$ & 306.20 & 105505.15 & 306200.00 & 200694.85 & $1: 1.92$ \\
\hline $\mathbf{T}_{\mathbf{3}}$ & 324.46 & 72779.96 & 324460.00 & 251680.04 & $1: 3.45$ \\
\hline $\mathbf{T}_{\mathbf{4}}$ & 288.13 & 78555.15 & 288130.00 & 209574.85 & $1: 2.66$ \\
\hline $\mathbf{T}_{\mathbf{5}}$ & 266.20 & 120930.13 & 266200.00 & 145269.87 & $1: 1.20$ \\
\hline $\mathbf{T}_{\mathbf{6}}$ & 268.40 & 85340.68 & 268400.00 & 183059.02 & $1: 2.14$ \\
\hline $\mathbf{T}_{\mathbf{7}}$ & 311.66 & 99722.39 & 311660.00 & 211937.61 & $1: 2.12$ \\
\hline $\mathbf{T}_{\mathbf{8}}$ & 271.80 & 101406.19 & 271800.00 & 170393.81 & $1: 1.68$ \\
\hline $\mathbf{T}_{\mathbf{9}}$ & 290.33 & 96827.77 & 290330.00 & 193502.23 & $1: 1.50$ \\
\hline
\end{tabular}

The increased availability of nitrogen, which is an important constituent of chlorophyll and protein thus causing more growth and favorable physical conditions of soil and availability of plant nutrients in sufficient quantities.

The present findings are in line with the results of (Reddy and Reddy, 2005) in onion, Mamta and Rao (2012) in brinjal, Kumar et al., (2014) in radish, Thanunathan et al., (1997), Shah et al., (2016) and Giraddi (1993). maximum leaf length $(22.49 \mathrm{~cm}$, $42.48 \mathrm{~cm}$ and $51.27 \mathrm{~cm})$, leaf diameter $(0.79 \mathrm{~cm}, 0.97 \mathrm{~cm}$ and $1.61 \mathrm{~cm})$ was recorded at different stages 50,100 and 150 days after transplanting by the application of FYM, Vermicompost and Neem cake $\left(\mathrm{T}_{7}\right)$ and Vermicompost $\left(\mathrm{T}_{2}\right)$.

This might due to the soil application of FYM might be due to the presence of $\mathrm{mg}$ might have helped in chlorophyll synthesis which in turn increase the rate of photosynthesis resulted in higher leaf lengthThe present findings are in line with the results of Sundharaiya et al., (2016) in onion, Premsekhar and Rajashree (2009) in okra and Kumari et al., (2011) in tomato.

\section{Yield attributing characters}

Significant variation was recorded among different treatments combinations for yield and its attributing traits (Table 4). In the present investigation, Maximum bulb length $(7.97 \mathrm{~cm})$, polar diameter $(6.52 \mathrm{~cm})$, Equatorial diameter $(7.01 \mathrm{~cm})$, neck length $(5.55 \mathrm{~cm})$, number of scales per bulb (11.06), Bulb weight $(48.67 \mathrm{~g})$, Yield per plot $(3.46 \mathrm{~kg} / \mathrm{ha})$ and Yield per hectare (324.46 q/ha) was recorded in treatment $\mathrm{T}_{3}$ (FYM). Neck thickness $(1.27 \mathrm{~cm})$ was recorded in treatment $\mathrm{T}_{7}$ (Vermicompost + FYM +Neem cake). This increased yield might be due to increased yield attributing characters like photosynthetic number of scales, polar and equatorial diameter. The present results are in conformity with the findings of Alkaff et al., (2002), Patil et al., (2005), Aswiniet al., (2005). It is relevant to note that, farmyard manure seems to be directly responsible in increasing crop yields either by accelerating the respiratory process by increasing cell permeability by hormone growth action or by combination of all these processes. It supplies nitrogen, phosphorus and potassium of which phosphorus is involved in cell division, photosynthesis and metabolism of 
carbohydrates whereas potash regulates proper translocation of photosynthates and stimulates enzyme activity which in turn might have increased the rate of growth and positive development in yield characters leading to high bulb yield of onion. Chetna et al., (2015) and Shah et al., (2016). Maximum total soluble solids $\left(12.30{ }^{0} \mathrm{~B}\right)$ recorded in treatment $\mathrm{T}_{3}(\mathrm{FYM})$.

\section{Economics}

The perusal of data pertaining to cost benefit ratio (Table 5) revealed that maximum $\mathrm{C}$ : $\mathrm{B}$ ratio $(1: 3.45)$ was obtained with the treatment $\mathrm{T}_{3}$ (FYM) due to higher yield and lower cost of cultivation. These results are in line with findings of Premsekhar and Rajashree (2009) in okra.

On other hand, minimum C.B ratio $(1: 1.20)$ was obtained with the $T_{1}$ (control) due to reduced yield, lesser gross income ultimately lesser net return. Hence, taking into consideration all aspects, treatment T3 (FYM) due to its persistent performance for yield (324.46q/ha), gross income (Rs.324460.00), net returns (Rs.251680.04) and higher cost benefit ratio (1:3.45). Hence, treatment $\mathrm{T} 3$ (FYM) can be recommended for the cultivation of onion.

In conclusion, among the different treatments applied best Growth and Yield parameters were found from the plants grown in the plots applied with Vermicompost and FYM respectively whereas, some of the parameters viz. Leaf diameter Leaf length and neck thickness were seen superior in the combination of FYM + Vermicompost + Neem cake. Under organic cultivation of onion, maximum Cost Benefit ratio was seen in Farmyard manure i.e., 1:3.45 with a total net return of Rs. 251680.04. Thus, it can be concluded that use of FYM $\left(2.8 \mathrm{~kg} / 1.2 \mathrm{~m}^{2}\right)$ can be recommended for the cultivation of onion.

\section{Highlight}

- $\quad$ FYM at the rate of $2.8 \mathrm{~kg} / 1.2 \mathrm{~m}^{2}$ can be recommended for cultivation of onion in hilly region of Uttarakhand.

- FYM solely can produce $\mathrm{C}: \mathrm{B}$ ratio greater than one and more net return per unit area.

Acknowledgements: We take this opportunity to express our deep sense of gratitude, indebtedness and sincere thanks to all the field workers and plant breeding laboratory who helped during entire research work.

\section{References}

Alkaff, A.H., Saeed and Salim, A.Z. (2002). Effect of biofertilizer, inorganic, organic and foliar application of Power 4 on the productivity of onion. Univ. Aden. J. Natural Appl. Sci. 6(1): 1-14

Aswani, G., R. Paliwal, and D. K. Sarolia (2005).Effect of nitrogen and biofertilizer on yield and quality of rabi onion (Allium cepa L) cv. Puna Red. Agric. Sci. Digest. 25(2): 124-126

Bhawalkar, U.S (1991). Vermiculture biotechnology for low external input and sustainable agriculture-seminar on low external input sustainable agriculture. Amsterdam Netherlands pp. 1-6

Chetna, B., N. Shukla, K. P. Sharma, M. Patanwar, and D. Chandravanshi (2015). Effect of organic substances on yield and quality of onion, (Allium cepa L.). Int. J. of Farm Sci., 5(1): 30-35

Giraddi, R.A. (1993). Vermiculture and role in agriculture. In Course on the Officers of the State Department of Agriculture, Karnataka, and 18-20 October 1993 by the Department of Agricultural.

Gomez, K.A., and A.A. Gomez (1984) Statistical procedures for agricultural 
research. John Wiley and Sons Inc., New York. pp. 357-427.

Kumari, R., I. Kaur, A. K. Bhatnagar (2011). Effect of aqueous extract of Sargassumjohnstonii Setchell\& Gardner on growth, yield and quality of Lycopersicon esculentum Mill. J. of Appl. Phyco. 23: 623-633.

Kumar, S., S. Maji, S. Kumar, and H. D. Singh (2014). Efficacy of organic manures on growth and yield of radish (Raphanus sativus L.) cv. Japanese white. Int. J. of Plant Sci. 9(1): 57-60.

Mamta, K.A. Wani, and R. Rao (2012). Effect of vermicompost on growth of brinjal plant (Solanum melongena) under field conditions. J. New Biol. Rep. 1(1): 2528.

Patil, P.V., P.B. Chalwade, A.S. Solanke, and V.K. Kulkarni (2005). Effect of fly ash and FYM on nutrient uptake and yield of onion. J. of Soils and Crops. 15(1): 187-197.

Premsekhar, M. and V. Rajashree (2009). Influence organic manures on growth, yield and quality of okra. J. Sustain. Agric. 3(1): 6-8.

Ramesh, P., N. R. Panwar, A. B. Singh, S. Ramana, S. K. Yadav and A. S. Rao
(2010). Status of organic farming in India. Curr. Sci. 98(9): 1190-1194.

Reddy, K. C. and K. M. Reddy (2005) Differential levels of vermicompost and nitrogen on growth and yield in onion (Allium cepa L.) - radish (Raphanus sativus L.) cropping system. J. res. ANGRAU33(1): 11-17.

Shah, K. N., V. Singh, D. K. Rana (2016) Effect of organic and inorganic manures on growth, yield and quality of onion Cv. 'PushaMadhvi. J. Hort Flora Res. Spectrum, 5(3):233-237.

Sundharaiya, K., K. Sujatha, P. R. Renganayaki, G. Sathish (2016) Exploitation of organic inputs for growth and yield of multiplier onion (Allium cepa var. aggregatum) var. co (on 5). J. Prog. Agric. 7(2): 52-58.

Thamburaj, S. and Singh, N. 2001. Textbook of vegetables, Tuber crops and spices. ICAR. New Dehli, 469p.

Thanunathan, K., S. Natarajan, R. Senthilkumar and Arulmurugan, K. (1997) Effect of different sources of organic amendments on growth and yield of onion in mine spoil. J. Madras Agric. 84(7): 382-384.

\section{How to cite this article:}

Kalpana Nirala, Shailaja Punetha, S.C. Pant and Sandeep Upadhaya. 2019. Effect of Various Organic Manures with Biofertilizers on Growth, Yield and Economics of Onion (Allium cepa L.). Int.J.Curr.Microbiol.App.Sci. 8(01): 3092-3099.

doi: https://doi.org/10.20546/ijcmas.2019.801.330 